\title{
Taste Responsiveness, Weight Loss, and the Ponderostat
}

\author{
RICHARD E. NISBETT \\ Department of Psychology, University of Michigan, Ann Arbor, Michigan 48106 \\ AND \\ L. REID HANSON, JR., ALEXANDER HARRIS AND ADRIAN STAIR \\ Yale University, New Haven, Connecticut 06520
}

(Received 27 November 1972)

\begin{abstract}
NISBETT, R. E., L. R. HANSON, JR., A. HARRIS AND A. STAIR. Taste responsiveness, weight loss, and the ponderostat. PHYSIOL. BEHAV. 11(5) 641-645, 1973.-Rats which lost weight due to exercise showed increased acceptance of good tasting food and increased rejection of bad tasting food. This indicates that energy deficit results in heightened taste responsiveness and suggests that obese human beings may be highly taste responsive because they chronically maintain their weight at a level below its set point.
\end{abstract}

Taste responsiveness Exercise Obesity Ponderostat Glucostat Lipostat Food deprivation

JACOBS and Sharma [8] have recently made the surprising suggestion that hunger makes the animal highly responsive to the taste of food and relatively unresponsive to the state of its gut. They have shown that when the dog or rat is maintained on a $23 \mathrm{hr}$ deprivation schedule and allowed only one brief opportunity each day to eat laboratory chow, it eats about the same amount in the brief daily meal that it would eat in a $24 \mathrm{hr}$ period if allowed constant, ad lib access to laboratory chow. If the chow is made particularly good-tasting by the addition of fats or sugar, however, the hungry animal eats more in the brief meal than it would eat over a $24 \mathrm{hr}$ period of ad lib access. Conversely, if the chow is made bad-tasting by the addition of quinine or cellulose, the hungry animal eats less food in its brief meal than it would eat over a $24 \mathrm{hr}$ period of ad lib access. In similar research, Gross [7] subjected young rats to deprivation schedules so severe that normal weight could not be attained. The animals were then returned to ad lib feeding routines and their food intake compared with that of normal weight animals always maintained on ad lib routines. The previously semistarved animals were much more responsive to the taste of food than the nondeprived animals, increasing their intake more than nondeprived animals when offered particularly good tasting food and decreasing their intake more when offered bad tasting food.

It is possible to interpret earlier research on saccharin preference in terms of the Jacobs and Sharma hypothesis. Bacon, Snyder, and Hulse [1] showed that, in the rat, food deprivation leads to an increase in preference for saccharin solutions over water. Valenstein [11] showed that, while nondeprived rats prefer moderately sweet glucose solutions to highly sweet saccharin solutions, food deprived rats actually prefer the highly sweet, nonnutritive saccharin to the less sweet, but nutritive, glucose solutions. These data are consistent with the notion that energy deficit produces heightened taste responsiveness.

Cabanac [2] has independently proposed a similar hypothesis. He suggests that, when the organism is below its set point for weight, this fact is detected by a ponderostat. The ponderostat suppresses normal physiological responses to short term changes in nutritional conditon and produces exaggerated responses to the taste of food. The proposal is supported by experiments with humans. Fasting subjects were asked to taste sweet solutions and report on their pleasantness [5]. Then they were asked to drink $50 \mathrm{~g}$ of glucose. Prior to drinking the glucose load, subjects reported that the sweet solutions had a pleasant taste. Following the glucose load, subjects reported that the sweet solutions no longer had a pleasant taste. However, after subjects were restricted to a diet resulting in a $10 \%$ weight loss over a period of weeks, the sweet solutions remained pleasant tasting even after consumption of glucose load [4]. Thus subjects became highly taste responsive during the period of energy deficit. Internal changes sufficient to make sweet tastes undesirable to the subject whose weight was at set point had no effect on the semistarved subjects.

These proposals are of more than theoretical concern, since they may shed some light on the state of obesity.

\footnotetext{
' The authors are indebted to Terry Powley for helpful commentary and criticism. This research was supported by NSF Grant 6B33918.
} 
Obese subjects behave like semistarving subjects, continuing to like the taste of sweet solutions after the glucose load [3]. Cabanac suggests that this is true because the obese person, responding to social pressure to lose weight, attempts to maintain his weight below its biologicallyestablished high set point. Nisbett [10] has noted that many other parallels exist between hungry humans and obese humans. Both are hyperemotional, highly inactive, and hyposexual. Both have high levels of free fatty acids in the blood, signifying energy deficit. And the eating behavior of the obese human resembles that of hungry organisms in several ways, including marked acceptance of good tastes and marked rejection of bad tastes.

The assumption that obese humans are hungry, however, is weakened by the fact that in the research on hunger, the state of energy deficit has been produced by acute, usually total, food deprivation. If obese individuals are indeed hungry their energy deficit would not be of this type except in rare cases of starvation dieting. Energy deficit in obese humans would instead be the result of chronic, long-term failure to eat cnough to maintain weight at its high set point. It would be useful to create in animals an analogy to this kind of modest, chronic energy deficit by maintaining weight at a level slightly below normal, and to examine the behavioral response to this kind of energy deficit.

Mayer and his colleagues [9] have shown that when a rat is exercised for an hour or so a day, it does not eat enough to compensate for the extra energy expenditure. Instead it decreases its food intake and maintains its weight at a lower level throughout the period of exercise. It is not known why the rat behaves in this way, but it is a quite useful phenomenon for present purposes. It is possible to ask whether such a chronic, mild, voluntary depletion of the fat stores will be sufficient to alter the eating behavior of the rat in a direction characteristic of obese humans and starving organisms.

\section{EXPERIMENT}

\section{Method}

Thirteen seven-month-old male Holtzman rats, weighing an average of $537 \mathrm{~g}$, were exercised for an hour each day in separate motor-driven activity wheels. The speed at which animals were required to move (approximately $36 \mathrm{ft}$ per minute) can best be described as a brisk walk. Thirteen control animals were placed in the apparatus each day but were not exercised.

Animals were allowed access to food for $22 \mathrm{hr}$ a day. Taste responsiveness was measured by comparing intake of Purina lab chow with intake of a highly preferred fat mixture composed of $55 \%$ Crisco shortening by weight and 45\% Purina lab chow. Animals were given a choice between the two foods for a three day period one week before exercise began and for a three day period one week after termination of exercise. A one day test was given after five days of exercise and another after maintenance for forty days on the exercise regimen. During the maintenance period rats were exercised only five days a week.

\section{Results}

Figure 1 presents intake by experimental and control animals of chow and high fat mix before, during, and after the exercise regimen. Prior to exercise, experimental and control animals consumed nearly identical proportions of chow and mix. After five days of exercise, when experimen- tal animals weighed $15 \mathrm{~g}$ less than control animals, experimental animals were slightly more taste responsive, consuming a significantly greater proportion of their intake in the form of the high fat diet $(p<0.02)$. (All $p$ values are based on two-tailed tests.) Experimental animals consumed less total food by weight than control animals, but the difference is typical of relative intakes during the exercise regimen. At the second one day test, after 40 days of the exercise regimen, experimental animals weighed $21 \mathrm{~g}$ less than control animals and were much more taste responsive than controls. Experimental animals consumed only $55 \%$ as much chow as control animals $(p<0.05)$ but consumed $17 \%$ more high fat mix than controls $(p<0.001)$. Exercised animals not only consumed proportionally more high fat food than control animals; they also increased their total caloric intake over baseline chow levels significantly more than did control animals. Exercised animals ate fewer calories than controls on the days prior to the oil mix test day, but consumed more total calories on the test day ( $p$ contrasting test day calories with preceding day's calories $<0.05)$.

At one week after the termination of exercise, experimental animals had regained $9 \mathrm{~g}$ of the weight differential. At this time they were only trivially more taste responsive than control animals.

The evidence thus indicates that exercise-induced weight loss results in a greater preference for high fat diets, with strength of preference being directly related to extent of weight loss. The possibility exists, however, that it is not the taste properties of the high fat diet which are relevant, but its greater caloric density or other nutritive properties. In order to demonstrate that exercise-induced weight loss results in greater response to taste per se, Experiment 2 was conducted using non-nutritive saccharin and quinine additives.

\section{EXPERIMENT 2}

\section{Method}

Two different taste manipulations, neither of which involved caloric changes, were employed in Experiment 2. In the first of these, rats were given access to both water and $0.325 \%$ saccharin solution. Both solutions and chow were available $22 \mathrm{hr}$ a day. The animals were the same rats employed in Experiment 1. After the weight of previously exercised animals had plateaued (experimental animals remained $4 \mathrm{~g}$ lighter than controls), baseline preferences for saccharin were obtained for a four-day period. Then the exercise regimen of Experiment 1 was reinstituted for the same 13 animals which had previously been exercised, and saccharin preference was studied during the first eight days of the regimen.

Response to bad tasting food was also examined. Five days after the end of saccharin-preference testing, the animals' chow was replaced with $0.5 \%$ quinine-adulterated chow for a period of three days.

\section{Results}

The effect of initiation of exercise was to slightly increase saccharin preference. Experimental and control animals displayed very similar preferences for saccharin during the base period $(p>0.25)$. Average daily consumption of saccharin for the last two baseline days was $31.16 \mathrm{cc}$ for experimental animals and $33.51 \mathrm{cc}$ for control animals. Water consumption was $29.96 \mathrm{cc}$ for experimental animals 


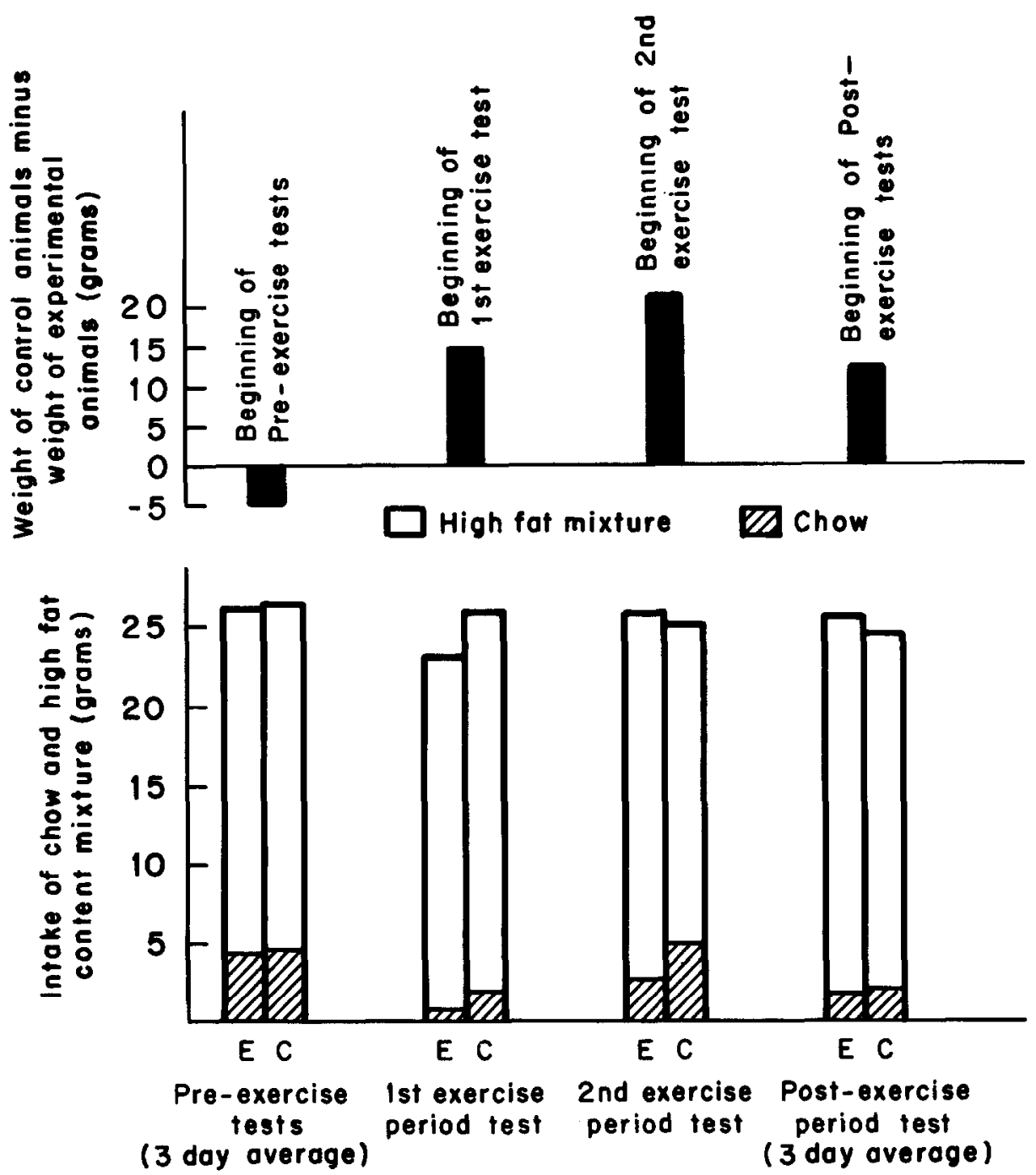

FIG. 1. Chow and high fat mixture eaten by exercised and control animals.

and $27.67 \mathrm{cc}$ for control animals. Saccharin preference of the experimental animals increased steadily over the first six days of exercise and then, coincident with the cessation of weight loss, abruptly reverted to baseline levels (see Fig. 2). However, the changes from baseline levels manifested by experimental and control animals did not differ significantly on any single day. It was only when data were grouped into two-day averages to provide stability to the highly variable saccharin preference scores that statistical significance could be demonstrated. When this was done, experimental animals were found to have increased their preference over baseline levels significantly more than control animals on the second $(p<0.05)$ and third $(p<0.025)$ of the four twoday blocks. The reversal on the fourth two-day block is not significant because baseline differences between experimental and control groups are in the same direction as differences in this period ( $p>0.40$ for the comparison between experimental and control differences from baseline levels).

Since the saccharin preference data were rather weak, the testing procedure was repeated at termination of exer- cise and preferences were observed during the subsequent weight gain period. On the day of termination of exercise, experimental saccharin preferences were not significantly higher than those of control animals. The difference between experimental and control elevations over baseline was significant for each of the next two days, however (both $p_{s}<0.025$ ). Differences between the two groups declined to insignificance thereafter. It thus appears that exercise-induced weight loss is associated with elevated saccharin preference and that preference differences disappear as weight gain progresses.

Figure 3 presents intake of chow and quinine adulterated chow. These tests were administered during the exercise regimen, five days after the first saccharin preference tests, at a point when experimental animals weighed $23 \mathrm{~g}$ less than controls. It may be seen that experimental animals consumed slightly (though nonsignificantly) less chow than control animals on the baseline day. Experimental animals then consumed considerably less quinine adulterated chow on each of the three days it was offered $(p<0.10$ on Day 1 , 


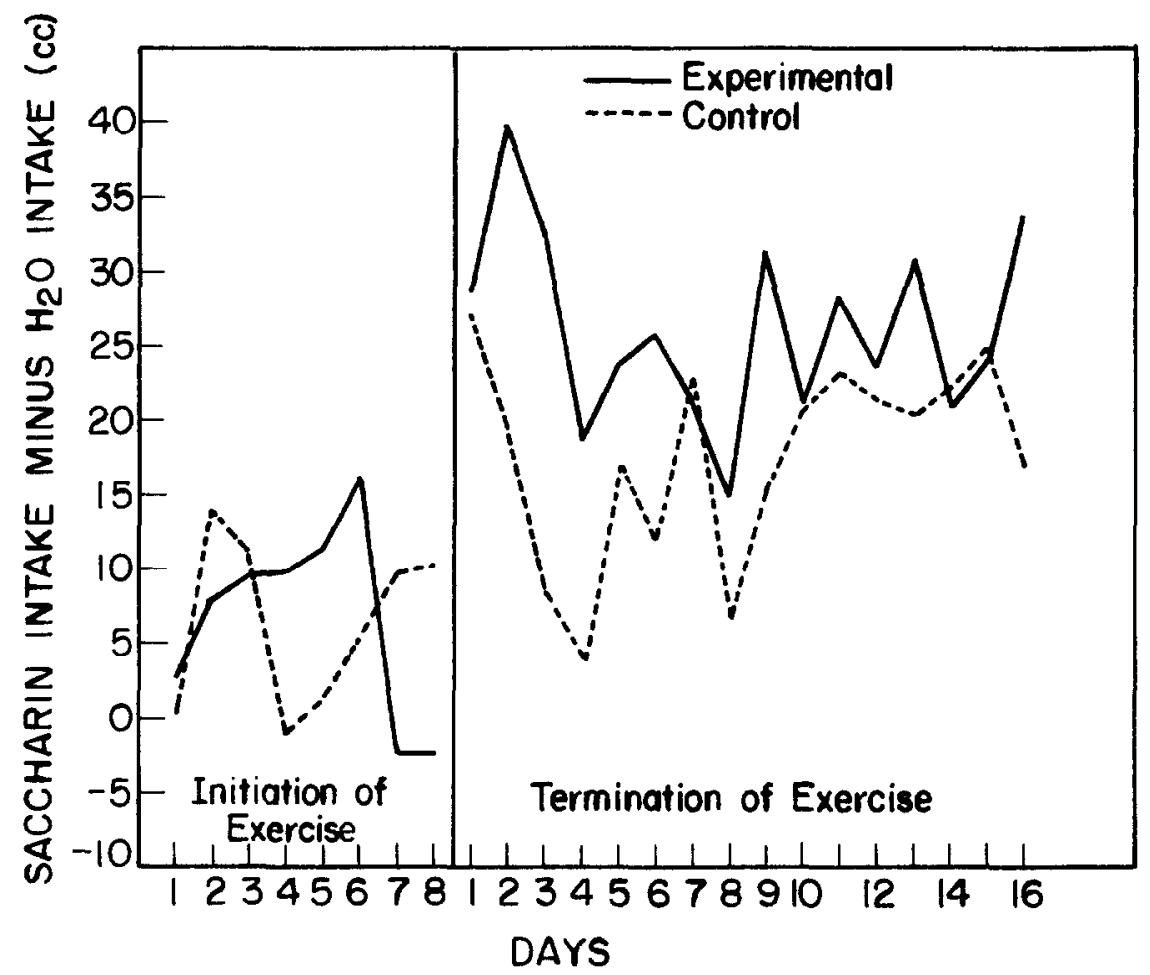

FIG. 2. Saccharin preference of exercises and control animals.

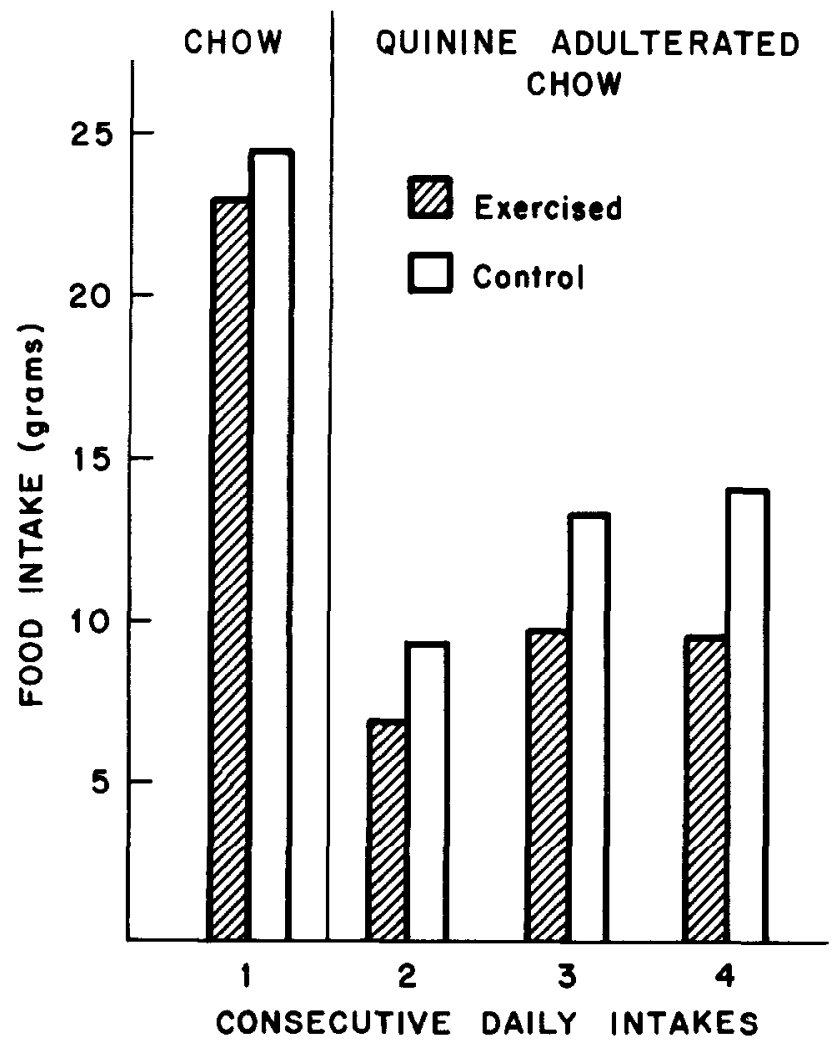

FIG. 3. Chow and quinine adulterated chow eaten by exerciscd and control animals.
$<0.01$ on Days 2 and 3). The food intake of experimental animals over the three day period of access to adulterated chow was a significantly smaller fraction of the baseline intake than that for control animals $(p<0.025)$. As a consequence, experimental animals lost a significantly greater proportion of their weight over the three day period $(p<0.05)$.

\section{DISCUSSION}

It might seem that the view of energy deficit proposed by Jacobs and Sharma, and supported by the present data, is at variance with common sense. The finding that exercised animals defend their lower weight less well than control animals defend their higher weight, when challenged with quinine adulterated chow, is particularly paradoxical at first blush.

However, it is possible to describe the Jacobs and Sharma position in such a way that common sense is not violated. It is not unreasonable to propose that, when the organism is in a state of chronic, long-standing energy deficit, physiological cues signalling short-term changes in nutritional state are ignored. If long term signals did not override short term signals, a semistarved animal presented with limitless opportunity to eat would never regain lost weight. The exaggerated response to taste might then be viewed as the result of the absence of any modulating influence of short term nutritional signals.

It should also be pointed out that the rejection of bad tasting food by the hungry animal might have adaptive significance. There might be a survival advantage for animals with a tendency to grow increasingly wary of bad tasting (and possibly poisoned or injurious) food when hungry. 
The exercise manipulation is viewed by the authors as a means of producing a drop from previously established set point and consequent energy deficit. But it should be noted that other interpretations of the manipulation are also possible. First, it could be that the real set point of the animal is its exercised weight, and that its unexercised weight represents an elevation above set point. If so, then it is a hyposensitivity to taste on the part of the sedentary, energysurfeited animal that has been demonstrated rather than a hypersensitivity on the part of the exercised animal. Obviously, some independent means of establishing true set point is needed. It is to be hoped that converging physiological and behavioral data will ultimately provide such an independent definition.

A second interpretation is quite orthogonal to any notion of energy deficit or surplus. Collier, Hirsch, and Leshner [6] have suggested that exercise initiates a highly efficient lypolytic mechanism which may not require as large an adipose tissue store for a given energy reserve as is required by the sedentary animal. If this is correct, then it may be more accurate to speak of exercise as lowering the body's set point for adipose mass rather than lowering adipose mass below the set point. Such an interpretation, it should be noted, would lessen the likely relevance of the present study to the data on the eating behavior of the obese human.

On the other hand, if it is weight change rather than altered lypolytic mechanism which is the chief consideration, then it would appear that a useful analogy to the dieting behaivor of obese humans has been demonstrated. The potential practical significance of the present results becomes obvious when weight loss of the rats in the present study is translated into human terms. Maintenance of a $537 \mathrm{~g}$ rat at $516 \mathrm{~g}$ is roughly comparable to a $200 \mathrm{lb}$ man holding his weight to $192 \mathrm{lb}$. If such a tranlation is valid, then it is quite possible that the eating behavior peculiarities of some obese humans may indeed be traceable to a tendency to hold their weight slightly below set point.

\section{REFERENCES}

1. Bacon, W. E., H. L. Snyder and S. H. Hulse. Saccharin preference in satiated and deprived rats. J. comp. physiol. Psychol. 55: $112-114,1962$.

2. Cabanac, M. Physiological role of pleasure. Science 173: 1103, 1971.

3. Cabanac, M. and R. Duclaux. Obesity: Absence of satiety aversion to sucrose. Science 168: 496-497, 1970.

4. Cabanac, M., R. Duclaux and N. H. Spector. Sensory feedback regulation of body weight: Is there a ponderostat? Nature 229: 125-127, 1971.

5. Cabanac, M., Y. Minaire and E. R. Adair. Influence of internal factors on the pleasantness of a gustative sweet sensation. Communs. behav. Biol. 1: 77-82, 1968.

6. Collier, G., E. Hirsch and A. I. Leshner. The metabolic cost of activity in activity-naive rats. Physiol. Behav. 8: 881-884, 1972.
7. Gross, L. P. Scarcity, unpredictability and eating behavior in rats. Unpublished Ph.D. Dissertaiton, Columbia University, 1968.

8. Jacobs, H. L. and K. N. Sharma. Taste versus calories: sensory and metabolic signals in the control of food intake. Ann. N. Y. Acad. Sci. 157: 1084-1125, 1969.

9. Mayer, J., N. B. Marshall, J. J. Vitale, S. H. Christensen, M. B. Mashayekhi and F. J. Stare. Exercise, food intake and body weight in normal rats and genetically obese adult mice. $\mathrm{Am}$. J. Physiol. 177: 544-548, 1954.

10. Nisbett, R. E. Hunger, obesity and the ventromedial hypothalamus. Psychol. Rev. 79: 433-453, 1972.

11. Valenstein, E. S. Selection of nutritive and nonnutritive solutions under different conditions of need. $J$. comp. physiol. Psychol. 63: 429-433, 1967. 